\title{
Trypanosomatid Aquaporins: Roles in Physiology and Drug Response
}

\author{
Goutam Mandal ${ }^{1, \dagger}$, Jose F. Orta ${ }^{1, \dagger}$, Mansi Sharma ${ }^{1,2}$ and Rita Mukhopadhyay ${ }^{1, *}$ \\ 1 Department of Cellular Biology and Pharmacology, Herbert Wertheim College of Medicine, \\ Florida International University, 11200 SW 8th Street, Miami, FL 33199, USA; \\ E-Mails: gmandal@fiu.edu (G.M.); jorta004@fiu.edu (J.F.O.) \\ 2 Department of Biological Sciences, Florida International University, 11200 SW 8th Street, Miami, \\ FL 33199, USA; E-Mail: mshar025@fiu.edu \\ $\dagger$ These authors contributed equally to this work. \\ * Author to whom correspondence should be addressed; E-Mail: rmukhop@fiu.edu; \\ Tel.: +1-305-348-1472.
}

Received: 2 December 2013; in revised form: 22 December 2013 / Accepted: 24 December 2013 / Published: 27 December 2013

\begin{abstract}
In the class Kinetoplastida, we find an order of parasitic protozoans classified as Trypanosomatids. Three major pathogens form part of this order, Trypanosoma cruzi, Trypanosoma brucei, and Leishmania, which are responsible for disease and fatalities in millions of humans worldwide, especially in non-industrialized countries in tropical and sub-tropical regions. In order to develop new drugs and treatments, the physiology of these pathogenic protozoans has been studied in detail, specifically the significance of membrane transporters in host parasites interactions. Aquaporins and Aquaglyceroporins (AQPs) are a part of the major intrinsic proteins (MIPs) super-family. AQPs are characterized for their ability to facilitate the diffusion of water (aquaporin), glycerol (aquaglyceroporin), and other small-uncharged solutes. Furthermore, AQPs have been shown to allow the ubiquitous passage of some metalloids, such as trivalent arsenic and antimony. These trivalent metalloids are the active ingredient of a number of chemotherapeutic agents used against certain cancers and protozoan parasitic infections. Recently, the importance of the AQPs not only in osmotic adaptations but also as a factor in drug resistance of the trypanosomatid parasites has been reported. In this review, we will describe the physiological functions of aquaporins and their effect in drug response across the different trypanosomatids.
\end{abstract}


Keywords: aquaporin; Aquaglyceroporin; drug resistance; Leishmania; Trypanosoma; Pentostam; antimony

\section{Introduction}

Trypanosomatid parasites are single celled pathogenic organisms considered a major cause of morbidity and mortality in tropical and subtropical regions worldwide. Pathogens of this order are the Leishmania species (causative agents of leishmaniasis), Trypanosoma brucei (African sleeping sickness), and Trypanosoma cruzi (Chagas disease). A total of 88 countries are reported to be endemic for leishmaniasis, with new case counts approximately 1.5 to 2 million per year [1]. Rapid increase of drug-resistant cases has further aggravated the situation [2]. Sleeping sickness threatens about 60 million people in 36 sub-Saharan Africa countries [3,4]. Fortunately, there is a recent trend toward a lesser number of reported cases [3]. However, patients with African trypanosomiasis are still a major challenge to clinicians, as diagnosis for central nervous system (CNS) involvement is problematic and treatment at CNS-stage can be highly toxic and fatal if left untreated. Chagas disease is a chronic parasitic infection that affects about 8 million people in the Latin American countries [5].

The trypanosomatid parasites undergo diverse and remarkably complex life cycles with interspecies transmission, frequently between an insect and a mammal, invasion of various cell types and/or extracellular growth in the blood stream, development of dormant stages in the mammalian host and differentiation to transmittable and infectious forms within the insect gut [6]. As they frequently travel between insect and mammalian hosts, they face severe osmotic challenges [7]. In the insect vector, the parasites differentiate into infective forms and migrate to the destined organ for successful perpetuation of the infection whereas within the mammalian host they need to find appropriate intra and/or extracellular milieu to evade the host defense system followed by disease progression. Several studies in other systems indicate that osmotic cues in the form of altering water and other solutes flux play a significant role in cellular morphogenesis and migration [8,9]. To respond to these osmotic cues all living cells require efficient regulation of these rapid water and solutes movements; however, the lipid bilayer membranes that encapsulate all living cells and several subcellular organelles are greatly impermeable to water and other polar solutes. Therefore, to overcome these indispensable biological barriers all living cells are obligated to employ membrane transporter(s) that will allow rapid and regulated movement of water and other non-polar solutes in and out of the cells and/or cellular compartments in response to extra- and/or intracellular signals. This puzzle baffled the scientific community for several decades until Peter Agre and co-workers demonstrated a channel-forming, integral membrane protein that facilitates water transport [10,11]. As the regulated movement of water is fundamental to all life process later studies reported the ubiquitous presence of the members of this channel protein [12]. These members are collectively called aquaporins (AQP). AQPs belong to MIPs (Major Intrinsic Protein), a super-family of integral membrane proteins. These small integral membrane channels can be functionally classified into two sub-groups, water specific transporters (orthodox aquaporins), and aquaglyceroporins, which allow transport of small-uncharged polar solutes such as glycerol, metalloids and urea [13,14]. The physiological roles of AQPs are more extensive: 
(i) several recent studies have suggested that some members of orthodox AQP are permeable to a variety of substrates including gases [15], ions [16], and (ii) some members of aquaglyceroporins are adventitious transporters of trivalent metalloids such as antimonite (SbIII) and arsenite (AsIII) [17]. Structurally, most aquaporins share a common topology of six trans-membrane helices and two half-helices that fold into the center of the channel forming an hour-glass structure [18].

Physiological functions and regulation of AQPs have been extensively studied in human and several other model systems. By far the most extensively studied parasitic protozoan AQP is from Plasmodium. Plasmodium expresses a single AQP. AQPs from several species of Plasmodium share a high degree of homology with each other, mammalian AQPs and glycerol facilitator (GlpF) of Escherichia coli. Plasmodium AQPs are permeable to water, glycerol, urea, and several other polyols. Although this AQP is not essential for the parasite survival, glycerol transport through this channel plays a significant role during gametocytes development inside the red blood cells [18]. Several aquaporins have been identified in trypanosomatid parasites, from three in Trypanosoma brucei, [19,20] to four in Trypanosoma cruzi [20,21] to five in Leishmania [14,20]. When compared to E. coli and mammalian aquaporins, parasite AQPs are generally more robust water transporters. It appears that most protozoan parasites aquaglyceroporins are bifunctional and conduct both water and glycerol at reasonable rates [18].

High potential chemotherapy targets can be found at the parasite-host interface [20,22]. Integral membrane proteins such as AQPs play an important role on the parasitic organism adaptations and survival during all of its life cycle stages, even more so at the parasite host interface. Accordingly, AQPs could be attractive drug targets and/or mediators of specific drugs such as arsenic and antimony [20]. In P. falciparum glycolysis-related metabolites such as methylglyoxal and dihydroxyacetone inhibited proliferation at $200 \mu \mathrm{M}$ and $3 \mathrm{mM}$, respectively [23]. Although the levels of sensitivity against these compounds are far from ideal for clinical studies, these show promise that cytotoxic AQP substrates can be used for treating parasitic infections. Metalloids, such as arsenic and antimony, permeability of AQPs have also been exploited to treat several human diseases for more than a century. Arsenical and antimonial compounds are still being used as the primary line of treatment against trypanosomiasis and leishmaniasis [24,25].

After the discovery of the first AQP by Peter Agre in 1992, knowledge about human and other mammalian AQPs has expanded enormously within 20 years. However, the physiological significance of parasitic protozoan, especially trypanosomatid AQPs and their role in drug response are still in its infancy. Therefore, in this review, we discuss the current understanding and roles of kinetoplastid AQPs in trypanosomatid parasites with respect to physiology and drug response.

\section{Leishmania spp.: Leishmaniasis}

\subsection{Life Cycle}

In order to thrive, leishmaniasis needs to complete a triad of complex interactions between the Leishmania parasites, the sand fly vector, and the mammalian host. Female sand flies of the genus Phlebotumus (Old World) and Lutzomiya (New World) are the most important vectors responsible for the transmission of the disease. Leishmania parasites change their morphology depending on their stage in life cycle. These distinctive morphologies are known as promastigotes and amastigotes. 
Promastigotes are extracellular and characterized for their slender spindle like body with an anterior flagellum, and reside in the sand fly gut. Promastigotes proliferate by binary fission. Once promastigotes are phagocytized by mammalian macrophages they are transformed into amastigotes, which appear as oval-shaped and aflagellated structures that reside inside phagolysosomes. Amastigotes, which also proliferate by binary fission, finally burst out of the infected cell to infect fresh macrophages and perpetuate the cycle. The life cycle of Leishmania starts when the sand fly ingests an infected blood meal from an infected host. After being ingested by the fly, amastigotes transform into promastigotes. Rapid morphological transformation and development of the parasite inside the vector is prompted due to the change in conditions (such as, increased $\mathrm{pH}$ and decreased temperature) between the mammalian host and the sand fly midgut. Initially, within the peritrophic vesicle amastigotes transform into weakly motile and dividing forms with a short flagellum at the anterior end of the parasite, known as procyclic promastigotes [26]. About 48 to 72 hours later, procyclics differentiate into non-dividing and highly motile nectomonad promastigotes that escape the peritrophic vesicle and move into the midgut lumen. Later at the anterior end of the midgut, nectomonads differentiate into proliferating leptomonads. Finally, leptomonads differentiate into two morphologically distinct forms: non-motile haptomonads promastigotes and highly motile with long flagellum metacyclic promastigotes, which are infective to mammalian hosts [27-29]. In its next blood meal the sand fly will inoculate the infectious metacyclis into the mammalian host. Transmission of cutaneous leishmaniasis (CL), mucocutaneous leishmaniasis (MCL) and visceral leishmaniasis (VL) caused by $L$. infantum and $L$. chagasi can be both zoonotic or anthroponotic whereas only anthroponotic transmission has been reported for VL caused by L. donovani [30].

\subsection{Disease}

The disease caused by Leishmania is called leishmaniasis. The disease is endemic to 88 countries in five continents. The majority of cases are reported from countries in the tropics and subtropics. Depending on the species the spectrum of disease can range from self-healing but disfiguring cutaneous leishmaniasis (CL) to more debilitating mucocutaneous leishmaniasis (MCL) to potentially fatal visceral leishmaniasis (VL). CL is caused by several species; in the Old World L. major and L. tropica are primarily responsible whereas in the New World L. mexicana, L. braziliensis, L. panamensis and L. amazonensis are the primary causative agents. MCL is endemic to the New World and caused principally by L. braziliensis. VL is reported to be caused by L. donovani (Asia and Africa), L. infantum (Europe) and L. chagasi (New World) [30-32]. Even though disease incidence is difficult to accurately assess, an estimate of 10 to 12 million people are infected worldwide with 350 million people at risk for transmission, and an annual approximate of 2 million new cases (about 1.5 million CL/MCL and rest VL) and over 50,000 deaths [33]. Additionally, current emergence of Leishmania/HIV co-infection is imposing a real threat in various parts of the world [34]. In CL parasites are confined to the dermal macrophages of the inoculation site(s) and inflict localized ulcerating but painless lesion(s). However, depending on the host immunological status the infection by L. aethiopica (Old World) and L. amazonensis (New World) can spread over large areas to cause diffused CL (DCL). Clinical presentation of DCL resembles lepromatous leprosy. [35,36]. CL caused by L. braziliensis usually takes longer time to heal compared to CL caused by other species in the New World. More 
importantly, in 1 to $3 \%$ of L. braziliensis infected patients, parasite can metastasize into mucous membrane of the naso-pharynx to develop MCL. The infection is usually chronic and metastatic spread often causes severe mutilation of soft tissues and cartilages [36]. Presence of a symbiotic RNA virus in L. braziliensis genome was reported to be associated with severity of the clinical outcome of MCL [37]. In VL, also known as kala-azar, parasites infect macrophages of the visceral organs primarily spleen, liver and bone marrow. VL is almost always fatal if left untreated. Incubation period of VL can vary from few months to several years. Initial skin lesion at the inoculation site is not apparent in VL and the onset of the disease is usually gradual with nonspecific symptoms, such as, general feeling of illness. Later, fever may occur occasionally. Once the disease is established, fever may occur more regularly with double or triple peak each day. Hepatosplenomegaly and inguinal lymphadenopathy along with occasional abdominal pain or abdominal discomfort are common clinical signs at this stage of the disease. At more advanced stages darkening of the skin color may happen [36,38]. Post-kala azar dermal leishmaniasis (PKDL) is a sequel of VL where the causative agent is L. donovani. PKDL can happen in up to $20 \%$ cases in Indian subcontinent and $>50 \%$ in Sudan after clinical cure of VL. In PKDL, the parasites become dermotropic and infect dermal macrophages. In India, PKDL may appear after years to decades of clinical cure of VL whereas in Sudan it appears within few months of cure. Except for cosmetic unattractiveness, PKDL usually does not cause any clinical complication or physiological discomfort. Consequently, many patients do not seek any treatment. This is an important factor in the epidemiological point of view as PKDL patients are potential human reservoir for the anthroponotic transmission cycle of L. donovani [38].

\subsection{Treatment and Drug Resistance}

Organic pentavalent antimonial compounds, stibogluconate (Pentostam) and meglumine antimoniate (Glucantime) have been the first line treatment against all forms of leishmaniasis for more than six decades. However, the clinical efficacies of these drugs are currently being challenged by the emergence of acquired resistance [39]. Indian subcontinent is a major contributor of VL. At present, more than $60 \%$ of Indian VL patients are unresponsive against antimonial treatment [40]. Pentavalent antimonials $[\mathrm{Sb}(\mathrm{V})]$ are pro-drugs and they are reduced to trivalent antimony [Sb(III)] - the active form of the drug (Figure 1) [41]. Whether the reduction of pentavalent to trivalent compound takes place either in the macrophages, in the parasite or in both is still unknown [42]. Entry mechanisms of antimonials into macrophages and phagolysosomes are yet to be identified. Nevertheless, Leishmania aquaglyceroporin 1 (LmAQP1), is the first $\mathrm{Sb}(\mathrm{III})$ facilitator identified in Leishmania (Figure 1) [14]. The second line of treatments includes amphotericin B, Alkyl-lysophospholipids (ALP) such as miltefosine and edelfosine, and pentamidine. Although miltefosine was originally developed as an antineoplastic drug, it also demonstrated significant antileishmanial activity. Miltefosine is the first oral antileishmanial drug approved in India. It has been successfully used against VL, including antimony unresponsive cases in India. However, adverse gastrointestinal, hepatic and renal side effects have been observed. In addition, contraindications with women in childbearing age along with high cost are major limiting factors. Even though clinical resistance to miltefosine has yet to be reported, it has been observed that after 9 to 12 months of successful treatment there is patient relapse, whether this is because of reinfection or developed resistance by the parasite it is yet to be studied [43]. 
Amphotericin B deoxycholate $(\mathrm{AmpB})$ is currently the first line of treatment against antimony unresponsive VL in India. Unfortunately, as in miltefosine, adverse side effects are major limiting factors of AmpB treatment. Although it has been shown that lipid formulations of amphotericin B are highly promising in respect to cure rate and toxicity, high cost of these lipid formulations is an impediment for mass scale use in the endemic countries such as India. More second line options, such as, allopurinol, atovaquone, fluconazole, paromomycin, and sitamaquine are in various stages of clinical trials [40].

Figure 1. Diagrammatic representation of proposed antimonial transport mechanism in a Leishmania infected macrophage. Pentavalent Antimony $[\mathrm{Sb}(\mathrm{V})]$ is transported into the macrophage, reduced to trivalent antimony [Sb(III)], and consequently transported into the phagolysosome. Finally, LmAQP1 facilitates the transport of Sb(III) into the parasite cytoplasm.

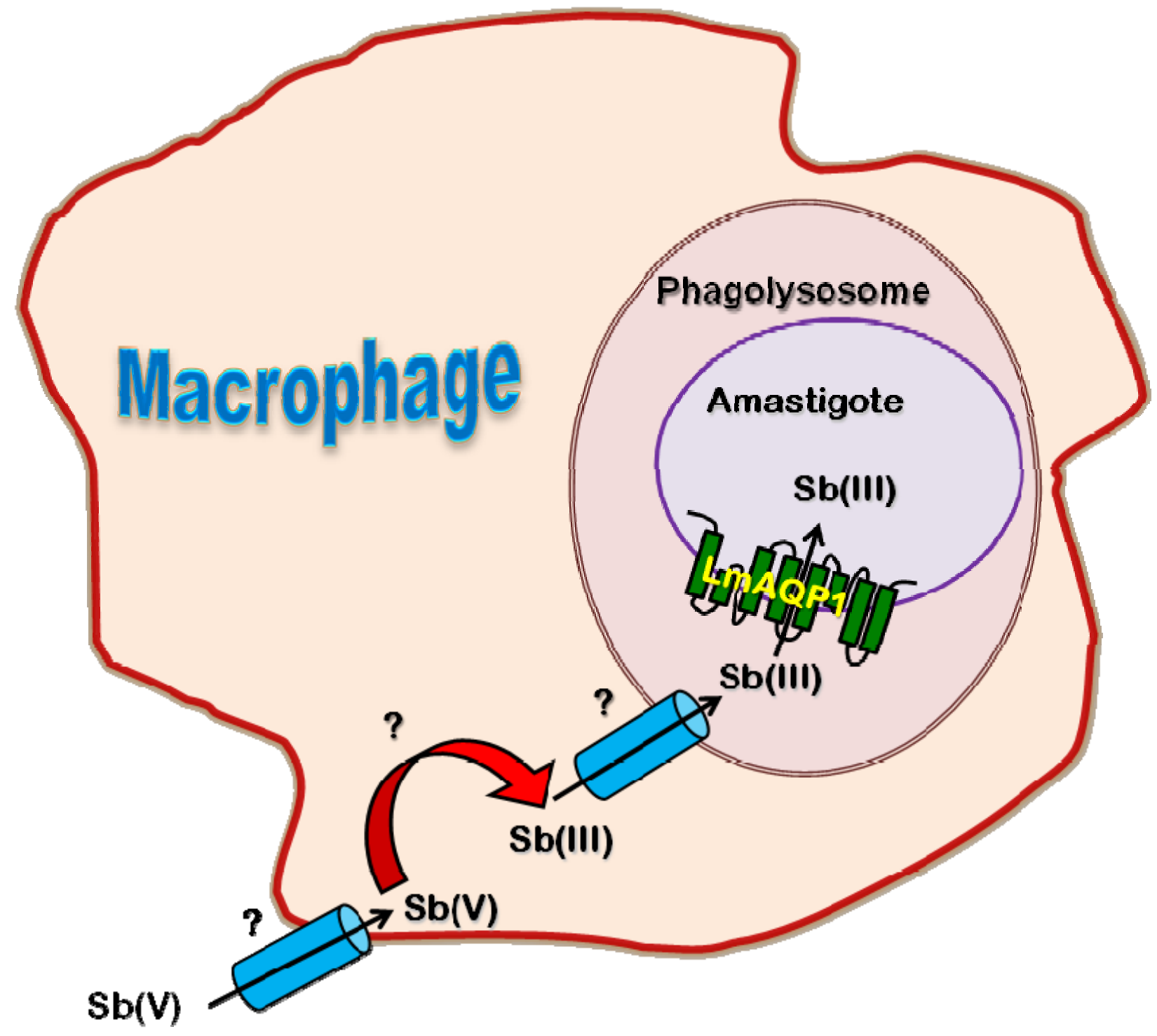




\subsection{Role of AQP in Parasite Physiology and Drug Response}

To date, LmAQP1 is the only reported Sb(III) transporter in Leishmania. Disruption of one of the two LmAQP1 alleles in Leishmania major resulted into a 10-fold increase in resistance to $\mathrm{Sb}$ (III) [44]. This has been corroborated by downregulation of LmAQP1 mRNA levels in clinical isolates of drug resistant L. donovani $[45,46]$. On the other hand, over expression of LmAQP1 makes the wild type parasites $>100$ fold more sensitive to $\mathrm{Sb}(\mathrm{III})$. More importantly, overexpression of LmAQP1 can reverse the phenotype in drug resistant isolates [47]. Taken together, LmAQP1 plays a significant role for the successful treatment of leishmaniasis using antimonials. Other factors such as levels of trypanothione, antimonite reductase and antimony-TSH conjugate exporter also play an important role in generation of drug resistance phenotype [48]. The L. major genome encodes five aquaporins: LmAQP1, LmAQP $\alpha, \operatorname{LmAQP} \beta, \operatorname{LmAQP} \gamma$, and LmAQP $\delta$. While LmAQP1 shows strong similarity to bacterial aquaglyceroporin GlpF and human AQP9 the other L. major aquaporins (LmAQP $\alpha-\delta$ ) are closer to plant aquaporins [18]. LmAQP1 is an avid water transporter, and the conduction capacity of this aquaglyceroporin is $65 \%$ of that of human AQP1. In contrast to Plasmodium and Trypanosome AQPs, which are inhibited by mercurials, LmAQP1 is a mercurial independent channel. LmAQP1 also helps in the conduction of glycerol, glyceraldehydes, dihydroxyacetone (DHA) and sugar alcohols. Expression of LmAQP1 is limited to the flagellum of promastigotes whereas in amastigotes it is localized to the flagellar pocket, the rudimentary flagellum and the contractile vacuoles [47]. Furthermore, LmAQP1 plays a major role in volume regulation and osmotaxis, two important factors to overcome the osmotic challenges the parasite faces during its life cycle [47]. Therefore, it is evident that LmAQP1 plays an important role in Leishmania physiology and drug resistance. At physiological $\mathrm{pH}$, the trivalent metalloids behave as molecular mimics of glycerol, and are conducted through aquaglyceroporin channels. The extracellular loop C of LmAQP1, which connects the adjacent transmembrane helices 4 and 5 of the protein, plays an important role in solute selectivity and function of the channel. In the absence of crystal structure, our structure-function studies have identified the critical residues of loop $\mathrm{C}$ for channel selectivity and function of LmAQP1. We identified that glutamate152 of loop $\mathrm{C}$ is responsible to discriminate between metalloids and glycerol [49]. Alanine 163 in loop $\mathrm{C}$ resides near the pore mouth and it is critical for the channel function, as alteration of this alanine may inactivate the channel function [50]. Expression of LmAQP1 in Leishmania is highly regulated. In absence of definitive promoters, Leishmania regulates its genes at post-transcriptional and/or post-translational levels. Recently, we reported that mitogen activated protein kinase 2 (MPK2) positively regulates the stability of LmAQP1 by phosphorylation at threonine 197. Dephosphorylation made LmAQP1 more susceptible to degradation. Similarly, an altered MPK2 that was incapable of phosphoryl transfer also destabilized LmAQP1 (Figure 2). Interestingly, phosphorylation at Thr197 also affected the localization of LmAQP1 and (Figure 2) altered the drug sensitivity and osmoregulatory activity of the parasite [51]. Altogether, LmAQP1 seems to play a major role in the ability of the parasite to cope with the osmotic challenges presented by the vector and host. Downregulation of LmAQP1 is also responsible for antimonial resistance. Hence it is tempting to propose that agonists and antagonists of LmAQP1 may be used to alter drug resistance and/or for transmission intervention respectively. 
Figure 2. Immunofluorescence of $L$. donovani promastigotes transfected with either wild type or altered LmAQP1 and/or LmMPK2. A) overexpression of LmAQP1, B) co-expression of LmAQP1 and MPK2, C) overexpression of T197A LmAQP1, D) co-expression of LmAQP1 and MPK2 K42A. Cells were stained for LmAQP1 (green), $\alpha$-tubulin (red); and DNA (blue). The three fluorescence images were finally merged.

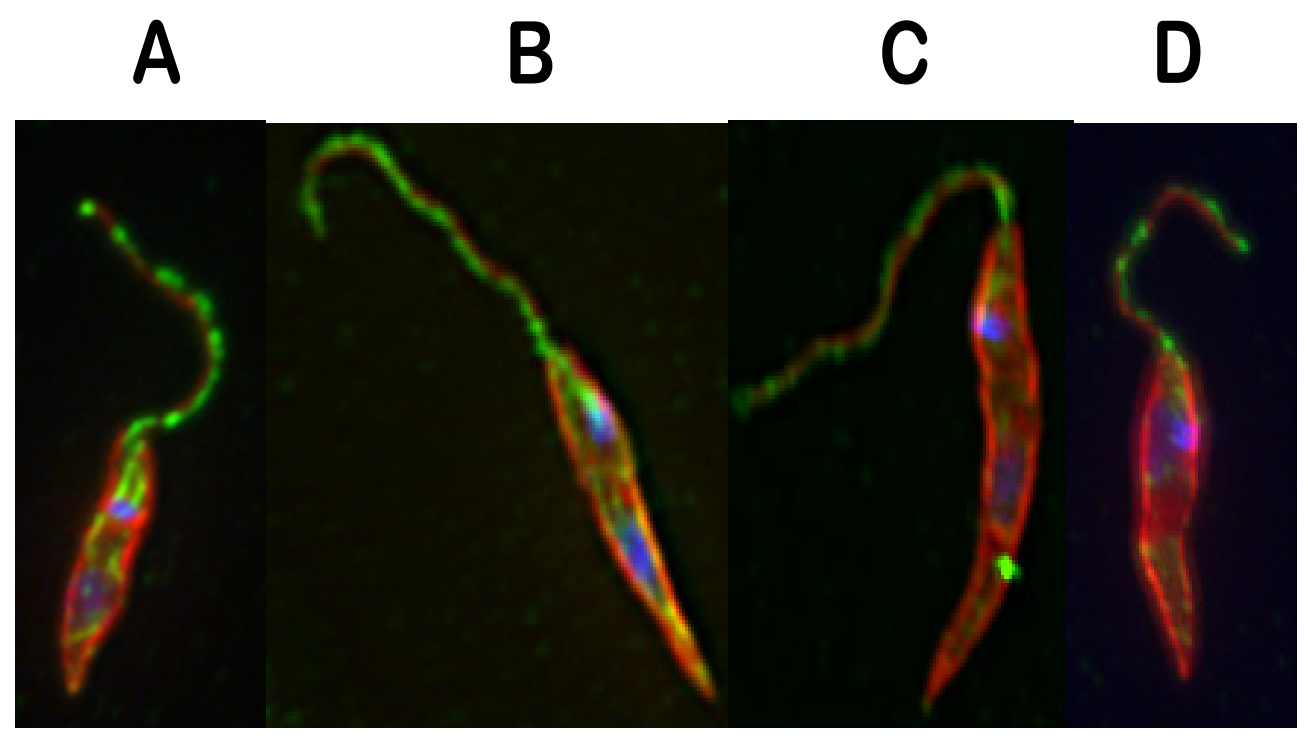

\section{Trypanosoma brucei: Human African Trypanosomiasis (HAT)}

\subsection{Life Cycle}

Human African Trypanosomiasis (HAT) also known as sleeping sickness is caused by Trypanosoma brucei. This vector-borne disease is endemic to the sub-Saharan Africa. The life cycle of T. brucei is complex and divided into insect vector stage and mammalian host stage. During blood feeding, an infected tsetse fly inoculates metacyclic trypomastigotes into the skin of the host. From the skin, parasites enter the bloodstream via lymphatic system and transform into elongated bloodstream trypomastigotes, which multiply by binary fission and can later invade central nervous system (CNS). Trypomastigotes are highly pleomorphic; they can range from slender bodied with long flagellum about $33 \mu \mathrm{m}$ in length to as short as $14 \mu \mathrm{m}$, stumpy and without free flagella. The stumpy forms with a rudimentary free flagella are non-dividing and cannot be transformed back to slender free flagellated form within mammalian hosts. When a fly feeds on an infected individual, only the stumpy non-free flagellated forms are transformed into dividing procyclic trypomastigote forms in the insect midgut. After approximately two weeks, procyclics migrate to the salivary glands. In the salivary glands, procyclics get attached to the epithelial cells and transform into dividing epimastigotes. After 2 to 5 days, some of the epimastigotes give rise to non-dividing stumpy metacyclic trypomastigotes, which are infective to mammals. The parasite takes about 3 weeks to complete the developmental cycle within the fly, and once infected the fly remains infective for the rest of its life [36]. 


\subsection{Disease}

HAT is eventually fatal if left untreated. Most of the cases are reported from poor and remote rural areas. HAT endemic zone has a strong correlation with the distribution of its vector, the tsetse fly (Glossina spp), which is endemic to the 37 sub-Saharan African countries. Approximately 60 million people are at risk but only about $15 \%$ are under surveillance [52]. The disease ranks at 7 th in the list 17 most neglected tropical diseases [53].

T. brucei has three morphologically identical sub-species- T. b. rhodesiense (East African or rhodesian HAT), $T$ b. gambiense (West African or gambian HAT) and $T$ brucei brucei (Animal African trypanosomiasis) [36]. The first two sub species can infect humans while $T$ b. brucei is not infective to humans. Depending on the growth phase of the parasite and the infested organs, the clinical symptoms of HAT are divided into two distinct and successive phases. At the beginning, growth of the parasite is restricted to the hemolymphatic system while at later stages parasites infiltrate into the CNS. The first phase symptoms are more acute and characterized by febrile lymphadenopathy and nonspecific hepatosplenomegaly and skin rashes. The second phase is more chronic, and patients develop meningoencephalitis with headaches and extensive neurological changes culminating into severe sleep disorders resembling narcolepsy, convulsions, and semi-coma eventually leading to death. The disease progression caused by $T b$. gambiense and $T b$. rhodesiense is not similar. Gambian HAT is characterized by a chronic progressive course with clear demarcation between phases and an average duration of around 3 years. On the contrary, rhodesian HAT is considered to be 'acute' as it represents with acute septico-pyaemia-like febrile illness and little phase demarcation that leads to fatality within months $[36,52,54,55]$.

\subsection{Treatment and Drug Resistance}

Treatment choices against HAT depend on the subspecies involved and the stage of the disease. At the hemolymphatic phase of infection pentamidine, an aromatic diamidine, and suramin, a colourless, polysulphonated symmetrical naphthalene derivative, are the drugs of choice [56]. However diagnosis is often late, especially in case of gambian HAT, and by the time of diagnosis the parasite has advanced to the CNS. HAT with CNS involvement is harder to treat because treatment choices are often limited by (i) inefficient penetrability across the blood brain barrier (BBB) and (ii) toxicity. Melarsoprol, a trivalent arsenical, and eflornithine, an ornithine analogue, or nifurtimox-eflornthine combination can reaches therapeutic levels inside the CNS [54].

Pentamidine is being used to treat HAT since 1930s and it is still the first choice of drug to treat the hemolymphatic phase of gambian HAT. A daily or alternate day deep intramuscular injection of $4 \mathrm{mg} \mathrm{kg}^{-1}$ body weight for total 7 to 10 injection is the recommended dosage regimen [57,58]. However the drug is ineffective against CNS stage of HAT because (i) serum binding and tissue retention reduce the availability of free drug to cross the $\mathrm{BBB}$ and (ii) even if a low amount of pentamidine cross the $\mathrm{BBB}$, it is taken out quickly by P-glycoprotein and multidrug resistance-associated proteins [57]. Pentamidine can be selectively accumulated inside the parasite cells up to millimolar levels. It primarily accumulates in the mitochondria followed by the nucleus and the acidocalcisomes. As a diamidine, pentamidine has high affinity for nucleic acid and it disrupts the mitochondrial 
genome especially the kinetoplast. High amount of pentamidine accumulation in the mitochondria also disrupts the mitochondrial membrane potential [57].

Use of pentavalent organic arsenicals as anti-trypanosomal agents dated back to 1900s. Less toxic melarsoprol with trivalent arsenic was introduced during 1940s. Since then it has been used constantly to treat HAT especially the late stage infection. Lipid solubility of melarsoprol formulation makes it permeable to BBB. It has been established that As(III) has strong affinity for glycerol-3-phosphate dehydrogenase, inhibit glycolytic enzymes and form a stable complex with trypanothione to form MelT thereby disrupting the energy and redox metabolism of the cells [4,56,59]. Although this drug is equally effective against both gambian and rhodesian late stage HAT, it is no longer the first line of treatment against gambian HAT primarily because of its toxicity, and reports of unresponsive cases due to drug resistance. However, melarsoprol is the only available option to treat CNS stage of rhodesian HAT [4]. Although the current incidence rate of HAT is promisingly decreasing [3], limited options for treatment along with prolonged use of same anti-trypanosomal agents have generated alarming levels $(20 \%-30 \%$ of cases in some areas) of melarsoprol unresponsiveness [57] and thus it could potentially revert back to a new epidemic form of HAT.

\subsection{Role of AQP in Parasite Physiology and Drug Response}

Only 3 AQPs have been identified and characterized in T. brucei namely TbAQP1, TbAQP2 and TbAQP3 [19,20]. TbAQP1 is localized in the flagellar membrane whereas TbAQP3 is localized in the pelicular membrane independent of the developmental stages [60,61]. Interestingly, TbAQP2 is confined to flagellar pocket in bloodstream forms and it is redistributed over the pelicular membrane in insect stage forms [61]. All characterized AQPs from T. brucei are aquaglyceroporins, they transport water along with other small uncharged molecules including glycerol, urea and trivalent metalloids $[19,62]$. Water and glycerol transport capacities for all TbAQPs are similar. It is presumed that as the blood stream form of $T$. brucei thrives under constant conditions in blood, it is never exposed to the osmotic challenges; however, when the parasites pass through renal medulla or are ingested by tsetse fly they face rapid changes in environmental osmolarity. Similarly, transmission from fly to mammals is also a massive osmotic insult [21,61]. Depending on the developmental stages $T$. brucei differentially regulates the abundance and localization of different AQPs [61]. Taken together, it is tempting to speculate that TbAQPs play critical roles to cope with these osmotic bottlenecks. Likewise, at the advance stage of $T$. brucei infection when parasite migrates to CNS, differential osmolite concentrations of blood and CSF (cerebrospinal fluid), especially low concentrations of glucose [63] glycerol [64] and urea [65] might send osmological cues via AQP for the navigation from blood to CSF. AQP plays significant roles in directional movements of cells as well as determining the cell shapes [12]. Several post-translational modifications of AQPs, such as, phosphorylation via protein kinases and altered cyclic adenosine monophosphate (cAMP) levels are known to modulate AQP localization thereby aiding their functionality and regulate osmotactic movements and cell shape [66-69]. T. brucei also experiences differential MPK activities and cAMP levels throughout their life cycle. For instance, TbMPK2 is necessary for developmental progression within the fly whereas TbMPK 5 down regulation and high level of cAMP promote blood stream stumpy form formation [70-72]. Differential localization of different TbAQPs at different lifecycle stages [60,61] may be related to these kinase 
mediated phosphorylation events. Additionally, transcript levels of different TbAQPs modulated depending upon the developmental stages of the parasite [60,61]. Taken together, it is highly likely that TbAQPs play significant roles (i) in directional movement of parasite from hind gut to salivary gland within the vector, (ii) changing the shapes depending on the developmental stages (iii) to cope with the osmotic insult, and (iv) in maintaining the glycerol homeostasis to avoid inhibition of glycolytic reaction.

Evidence of arsenical (atoxyl) unresponsive cases of HAT were reported back in the early 1900s by Paul Ehrlich who hypothesized that changes in specific 'chemioreceptors' could confer resistance. More recent studies have validated this hypothesis as alterations of several cell surface transporters could generate drug resistant phenotype [57,59]. Several physiologically important surface transporters could adventitiously facilitate drug uptake. In HAT, arsenical and diamidine resistant parasites are reported to be cross-resistant and have shown reduced uptakes of both drugs. Later studies have revealed that purine transporter $2(\mathrm{P} 2)$ facilitates the uptake of both arsenical and diamidine explaining the cross resistant phenotype (Figure 3). Uptake of both arsenicals and pentamidine are competitively inhibited by adenosine, the natural substrate of P2 (Figure 3). P2 is reported to be defective in both laboratory raised and clinical isolates of drug resistant parasites. However, the degree of resistance to pentamidine in a P2 null mutant of $T$. brucei was significantly lower when compared to both laboratory raised and clinical isolates of drug resistant parasites. More importantly, pentamidine or arsenical uptake by P2 null mutant was not inhibited in the presence of adenosine suggesting the presence of an additional high capacity transporter(s) for the drugs [57,59]. Recently, Alsford et al. (2012) identified the roles of TbAQP2 and TbAQP3 in pentamidine and melarsoprol cross-resistance by using high-throughput RNAi library screening method. TbAQP2/TbAQP3 null mutant cells are more than 2 fold and 15 fold resistant against melarsoprol and pentamidine respectively (Figure 3) [73]. Further studies revealed that TbAQP2 plays the pivotal role towards pentamidine and melarsoprol resistance. However, TbAQP2 does not play any role towards resistance against other arsenicals (such as, sphenylarsine oxide) or diamidines (berenil, phenanthridine trypanocides, isometamidium and ethidium) [59,61]. These suggest the existence of additional transporters. The role of TbAQP2 in pentamidine and melarsoprol cross-resistance was further substantiated by the discovery of altered TbAQP2, both in laboratory raised and in several clinical resistant isolates. In resistant isolates TbAQP2 locus was found to become either chimeric with TbAQP3 where $>120$ base pairs of 3' end of TbAQP2 was replaced by the nucleotide sequence of TbAQP3 3' end with concomitant loss of TbAQP3 alleles or deleted from the genome $[61,74]$. 
Figure 3. Melarsoprol and Pentamidine uptake mechanisms of T. brucei. T. brucei AQP2 and $\mathrm{P} 2$ facilitate the uptake of both melarsoprol and pentamidine. Adenosine competitively inhibit pentamidine uptake by P2. The HA1-3 (P type $\mathrm{H}^{+}$ATPase) might aid pentamidine uptake by providing a proton motive force to TbAQP2.

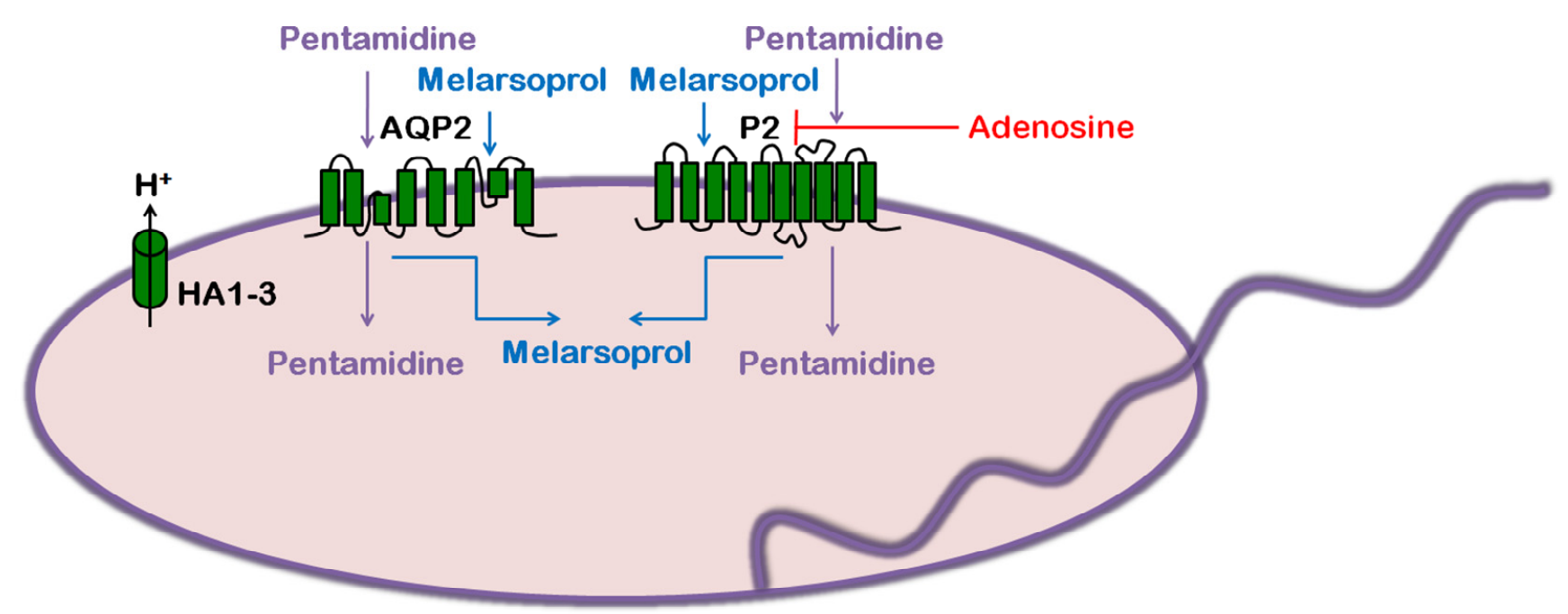

Dihydroxyacetone (DHA), a natural substrate of TbAQPs, can be used as an anti-trypanosomal agent. All 3 TbAQPs are highly permeable to DHA. DHA can be used as a carbon source by phosphorylating DHA to DHA phosphate (DHAP) using DHA kinase (DHAK). Later, DHAP can enter the glycolysis pathway. However, as DHAK gene is absent in T. brucei genome, they are unable to use DHA as a carbon source. Consequently, when T. brucei cells are exposed to DHA they can accumulate very high levels of DHA in the cytoplasm. High level of DHA causes cell cycle arrest at $\mathrm{G} 2 / \mathrm{M}$ phase followed by autophagic cell death. Although $\mathrm{IC}_{50}$ of DHA is rather high $(\sim 1 \mathrm{mM})$ for a drug candidate, it can serve as a potential starting point for the development of new analogs $[75,76]$.

\section{Trypanosoma cruzi: Chagas Disease}

\subsection{Life Cycle}

The life cycle of T.cruzi is complex and involves several developmental stages in its vector, a large sanguinivorous reduviid insect belonging to the subfamily of Triatominae, and in its hosts, about 150 species of wild and domestic animals including human. During the blood meal, the infected insect vector releases metacyclic trypomastigotes on the skin along with feces and urine. Once metacyclic trypomastigotes break inside the skin through the bite wound, they start invading the cells of local reticuloendothelial system and connective tissues, and differentiate into aflagellated dividing amastigotes inside the cells. After the host cell reaches its capacity, amastigotes transform back into trypomastigotes by growing flagella followed by lysis of the host cell [5,77]. The trypomastigotes are non-dividing but highly motile. Through the hemolymphatic system, they migrate to distant organs and primarily infect heart muscle, skeletal and smooth muscles, and ganglion cells to repeat the amastigote-trypomastigote cycle. Trypomastigotes have the capacity to invade any nucleated cell to form amastigotes. Circulating trypomastigotes are also infective to insect vectors. Along with the blood meal, trypomastigotes enter into the insect gut, and are transformed into proliferating 
epimastigotes. Later, epimastigotes move further down the gut to the rectum. At this stage of development, epimastigotes attach themselves to the rectal wall. Attachment of epimastigotes to the rectal wall induces metacyclogenesis. During the next blood meal the vectordefecates on the mammalian skin of the mammalian host, The metacyclic trypomastigotes in the feces enter the new host through the breaks on the skin [77,78]. Triatoma infestans, Rhodnius prolixus, and T. dimidiata are by far the most important vectors with regard to human Chagas disease. Triatomine ontogeny contains five nymphal stages followed by adults. All the developmental stages including adults are blood sucking, thus potentially can harbor and transmit T.cruzi. The disease can also be transmitted non-vectorially through blood transfusion, transplantation, transplacenta and even orally through the ingestion of contaminated food and drinks [36,79].

\subsection{Disease}

Chagas disease, also known as American trypanosomiasis, continues to affect human civilization since ancient times in many Latin American countries [80]. It is currently estimated that about 7 to 8 million people worldwide (primarily Latin America) are infected with Chagas disease [81] resulting into 10,000 to 14,000 deaths per year [77]. Presence of multiple vectors and reservoirs along with asymptomatic infection make the epidemiology of the disease more complex. Chagas disease is usually a lifelong disease [82]. The progression of the disease can be divided into three clinical phases: an acute phase and an intermediate phase followed by a chronic phase. The acute phase develops about 1 to 2 weeks after exposure to the pathogen. Acute phase infection is usually asymptomatic especially in adults. However, approximately $1 \%$ of patients develop nonspecific symptoms like fever, headache, anorexia, malaise, myalgia, joint pain, etc., with or without painful erythematous swelling called chagoma around the site of inoculation. Parasitemia is usually high in acute phase; trypomastigotes can be visible in blood smear. Every nucleated cell can be potentially invaded by the pathogen in the acute phase. After about 8 to 10 weeks, the majority of the patients start to develop antibodies against a variety of $T$. cruzi antigens. As a result, the parasitemia subsides significantly and spontaneously resolve the symptoms, but some patients may remain infected for years to decades without any symptoms. However, approximately $5 \%-10 \%$ of symptomatic cases can be fatal due to severe myocarditis, meningoencephalitis or both. Diminishing parasitemia leads to progression of the disease into the intermediate phase. In the intermediate phase, parasites are barely visible in the blood. Chronic phase of infection can develop in approximately 30\% of the patients, and most of them develop cardiomyopathy. Along with the heart disease, some patients may develop digestive system abnormalities, such as, megaesophagus and/or megacolon. The host immune status along with tissue damage inflicted during the acute phase of infection are believed to be the primary reason for chronic phase symptoms. In immunocompromised patients, Chagas disease may reactivate to develop more severe clinical symptoms $[5,36,83]$.

\subsection{Treatment and Drug Resistance}

Several drugs have been tried to treat the Chagas disease but effective treatment options are limited to only two drugs namely benznidazole and nifurtimox. Benznidazole and nifurtimox were introduced more than 4 decades ago. However, they are effective only against the acute phase of infection and 
contraindicated during pregnancy, and in patients with severe renal or hepatic inefficiency. The situation is further complicated by adverse side effects, such as, skin rashes, nausea, and kidney and liver failure [5,36,54]. Additionally, long treatment periods and high cost of the drugs promote treatment drop out which could potentially develop into drug resistance. An in vitro study reported the link between $T$. cruzi type I nitroreductase (NTR I) activity and cross-resistance against nufurtimox and benznidazole [84]. Another study proposed the possible association of NADPH-dependent cytochrome P450 reductase B (CPR B) and drug detoxification that could lead to unresponsiveness [85]. Recently, Campos et al (2013) reported that higher expression of P-glycoprotein efflux pump leads to drug resistance in $T$. cruzi [86].

\subsection{Role of AQP in Parasite Physiology and Drug Response}

Like other trypanosomatid parasites $T$. cruzi faces huge osmological fluctuations during its life cycle. The nature of the feeding cycle drives, the osmolarity of gut and the rectal content of triatomine bugs. Gut osmolarity varies between 300-970 mOsm whereas feces osmolarity ranges from 370 to $760 \mathrm{mOsm}$. On the contrary, osmolarity of mammalian blood is approximately $300 \mathrm{mOsm}$ [87]. As described earlier, AQPs are responsible to cope with these insults. Till date four AQP homologs namely, TcAQP and $\beta-\gamma$ have been identified in T. cruzi genome [18]. Among them, only TcAQP has been characterized so far. TcAQP is an orthodox aquaporin that transports only water and is sensitive to $\mathrm{HgCl}_{2}$ and $\mathrm{AgNO}_{3}$ inhibition. However, water conductance capacity of TcAQP is poor when compared to other parasitic AQPs. TcAQP expresses throughout all developmental stages of the parasite and is localized in the acidocalcisomes and contractile vacuole complex adjacent to the flagellar pocket of the parasites [21]. Acidocalcisomes are membrane bound acidic organelles that store calcium [88]. Acidocalciosomes are also present in some other unicellular organisms, such as, apicomplexan parasites (Plasmodium and Toxoplasma) [89], green algae Chlamydomonas reinhardtii [90], slime mold Dictyostelium discoideum [91], and agrobacterium Agrobacterium tumefaciens [92]. Acidocalcisomes and plant tonoplast have several functional similarities including osmoregulation [93]. Contractile vacuole is an established organelle involved in osmoregulation in protozoa. The basic structural plan of the contractile vacuole system is very similar in all protozoa. It consists of two parts: a central vacuole, or bladder, and a surrounding network of tubules and vesicles called spongiome. In T. cruzi acidocalcisomes and contractile vacuole system work together in a sequential manner to equip the parasite to face the hypoosmotic challenge during its transmission from vector to mammals. During hypoosmotic shock, a hypothetical adenyl cyclase is activated to form cAMP. Elevated cAMP level promotes fusion of acidocalcisomes with the contractile vacuole and translocation of an aquaporin to the contractile vacuoles, which aid them in water accumulation. Then, the water-loaded vesicles fuse with the flagellar pocket membrane to secrete the water (Figure 4) [7]. There is no report of association between any T. cruzi AQP and drug response. However, as hyposmotic shock is a major challenge for the parasite during transmission from the vector to the mammalian host, targeting osmoregulation systems for new drug development may be a feasible approach. 
Figure 4. Osmoregulation mechanism of T. cruzi. Volume increase due to hypo-osmotic stress triggers the production of cAMP. Elevated cAMP levels induce protein kinase activity that promotes fusion of acidocalcisomes (AC) with the contractile vacuoles (CV) and amino acid release. The fusion translocates the TcAQP from AC to CV and this is believed to aid the contractile vacuole in water sequestration followed by release into the flagellar pocket. AA: amino acids, PolyP: polyphosphate, Pi: inorganic phosphate.

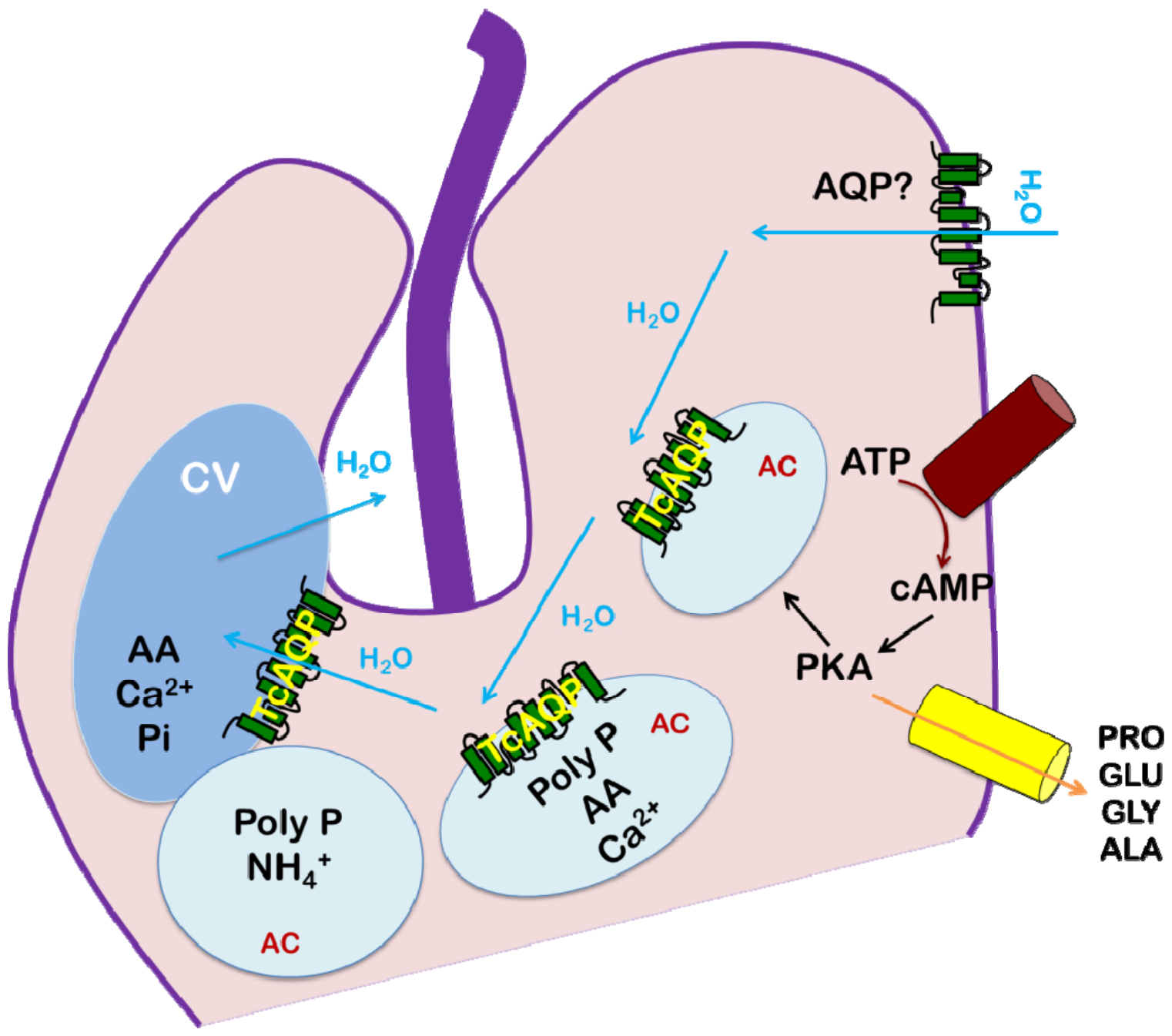

\section{Conclusions}

In the absence of effective vaccines against any trypanosomatid diseases, chemotherapy takes the center stage to combat them. However, increasing incidence of drug unresponsiveness, due to decades of use of the same or similar drugs, has created a major impediment. Second line choices are too limited, highly toxic and/or excessively expensive for these neglected diseases of the developing world. We are in dire need of cheaper and less toxic new drugs. Identification of parasite specific targets is critical for the development of new agents. Within two decades of discovery, significant advancement has been achieved with regard to mammalian AQPs. However, knowledge about AQPs from other living systems especially in parasitic protozoa is lagging far behind. Importantly, preliminary knowledge showed subtle but promising structural and functional differences between parasitic protozoan AQPs and their host counterparts. Parasite AQPs are necessary for the successful 
perpetuation of the diseases caused by them. In conclusion, further exploration of parasite AQP structures and functions including their regulatory mechanisms will eventually reveal their real potential for novel chemotherapeutic approaches and/or transmission intervention(s).

\section{Acknowledgments}

We acknowledge the startup funds from HWCOM, FIU to RM. MS is a Biomedical Research Initiative Fellow and JFO is a McNair Fellow at FIU for 2013.

\section{Conflicts of Interest}

The authors declare no conflict of interest.

\section{References}

1. den Boer, M.; Argaw, D.; Jannin, J.; Alvar, J. Leishmaniasis impact and treatment access. Clin. Microbiol. Infect. 2011, 17, 1471-1477.

2. Aït-Oudhia, K.; Gazanion, E.; Vergnes, B.; Oury, B.; Sereno D. Leishmania antimony resistance: What we know what we can learn from the field. Parasitol. Res. 2011, 109, 1225-1232.

3. Human African trypanosomiasis. http://www.who.int/trypanosomiasis_african/en/ (accessed on 27 November 2013).

4. Kennedy, P.G. Clinical features, Diagnosis, and treatment of human African trypanosomiasis (sleeping sickness). Lancet Neurol. 2013, 12, 186-194.

5. Rassi, A., Jr.; Rassi, A.; Marin-Neto, J.A. Chagas disease. Lancet 2010, 375, 1388-1402.

6. Gull, K. The biology of kinetoplastid parasites: Insights and challenges from genomics and post-genomics. Int. J. Parasitol. 2001, 31, 443-452.

7. Rohloff, P.; Docampo, R.A. Contractile vacuole complex is involved in osmoregulation in Trypanosoma cruzi. Exp. Parasitol. 2008, 118, 17-24.

8. Ishibashi, K.; Kondo, S.; Hara, S.; Morishita, Y. The evolutionary aspects of aquaporin family. Am. J. Physiol. Regul. Integr. Comp. Physiol. 2011, 300, R566-R576.

9. Verkman, A.S. Aquaporins at a glance. J. Cell. Sci. 2011, 124, 2107-2112.

10. Preston, G.M.; Carroll, T.P.; Guggino, W.B.; Agre, P. Appearance of water channels in Xenopus oocytes expressing red cell CHIP28 protein. Science 1992, 256, 385-387.

11. Chrispeels, M.J.; Agre, P. Aquaporins: Water channel proteins of plant and animal cells. Trends Biochem. Sci. 1994, 19, 421-425.

12. Loitto, V.M.; Karlsson, T.; Magnusson, K.E. Water flux in cell motility: Expanding the mechanisms of membrane protrusion. Cell Motil. Cytoskeleton 2009, 66, 237-247.

13. Borgnia, M.; Nielsen, S.; Engel, A.; Agre, P. Cellular and molecular biology of the aquaporin water channels. Annu. Rev. Biochem. 1999, 68, 425-458.

14. Gourbal, B.; Sonuc, N.; Bhattacharjee, H.; Legare, D.; Sundar, S.; Ouellette, M.; Rosen, B.P.; Mukhopadhyay, R. Drug uptake and modulation of drug resistance in Leishmania by an aquaglyceroporin. J. Biol. Chem. 2004, 279, 31010-31017. 
15. Musa-Aziz, R.; Chen, L.M.; Pelletier, M.F.; Boron, W.F. Relative $\mathrm{CO}_{2} / \mathrm{NH}_{3}$ selectivities of AQP1, AQP4, AQP5, AmtB, and RhAG. Proc. Natl. Acad. Sci. USA 2009, 106, 5406-5411.

16. Yool, A.J. Functional domains of aquaporin-1: Keys to physiology, and targets for drug discovery. Curr. Pharm. Des. 2007, 13, 3212-3221.

17. Engel, A.; Fujiyoshi, Y.; Agre, P. The importance of aquaporin water channel protein structures. EMBO J. 2000, 19, 800-806.

18. Song, J.; Mak, E.; Wu, B.; Beitz, E. Parasite aquaporins: Current developments in drug facilitation and resistance. Biochim. Biophys. Acta 2013, in press.

19. Uzcategui, N.L.; Szallies, A.; Pavlovic-Djuranovic, S.; Palmada, M.; Figarella, K.; Boehmer, C.; Lang, F.; Beitz, E.; Duszenko, M. Cloning, Heterologous expression, and characterization of three aquaglyceroporins from Trypanosoma brucei. J. Biol. Chem. 2004, 279, 42669-42676.

20. Beitz, E. Aquaporins from pathogenic protozoan parasites: Structure, Function and potential for chemotherapy. Biol. Cell 2005, 97, 373-383.

21. Montalvetti, A.; Rohloff, P.; Docampo, R. A functional aquaporin co-localizes with the vacuolar proton pyrophosphatase to acidocalcisomes and the contractile vacuole complex of Trypanosoma cruzi. J. Biol. Chem. 2004, 279, 38673-38682.

22. Kirk, K. Channels and transporters as drug targets in the Plasmodium-infected erythrocyte. Acta Trop. 2004, 89, 285-298.

23. Pavlovic-Djuranovic, S.; Kun, J.F.; Schultz, J.E.; Beitz, E. Dihydroxyacetone and methylglyoxal as permeants of the Plasmodium aquaglyceroporin inhibit parasite proliferation. Biochim. Biophys. Acta 2006, 1758, 1012-1017.

24. Mukhopadhyay, R.; Beitz, E. Metalloid transport by aquaglyceroporins: Consequences in the treatment of human diseases. Adv. Exp. Med. Biol. 2010, 679, 57-69.

25. Bhattacharjee, H.; Mukhopadhyay, R.; Thiyagarajan, S.; Rosen, B.P. Aquaglyceroporins: Ancient channels for metalloids. J. Biol. 2008, 7, 33.

26. Dostálová, A.; Volf, P. Leishmania development in sand flies: Parasite-vector interactions overview. Parasit. Vectors 2012, 5, 276.

27. Rogers, M.E.; Chance, M.L.; Bates, P.A. The role of promastigote secretory gel in the origin and transmission of the infective stage of Leishmania mexicana by the sandfly Lutzomyia longipalpis. Parasitology 2002, 124, 495-507.

28. Walters, L.I. Leishmania differentiation in natural and unnatural sand fly hosts. J. Eukaryot. Microbiol. 1993, 40, 196-206.

29. Kamhawi, S. Phlebotomine sand flies and Leishmania parasites: Friends or foes? Trends Parasitol. 2006, 22, 439-445.

30. Esch, K.J.; Petersen, C.A. Transmission and epidemiology of zoonotic protozoal diseases of companion animals. Clin. Microbiol. Rev. 2013, 26, 58-85.

31. Convit, J.; Castellanos, P.L.; Ulrich, M.; Castés, M.; Rondón, A.; Pinardi, M.E.; Rodríquez, N.; Bloom, B.R.; Formica, S.; Valecillos, L.; et al. Immunotherapy of localized, Intermediate, and diffuse forms of American cutaneous leishmaniasis. J. Infect. Dis. 1989, 160, 104-115.

32. Convit, J.; Ulrich, M.; Fernández, C.T.; Tapia, F.J.; Cáceres-Dittmar, G.; Castés, M.; Rondón, A.J. The clinical and immunological spectrum of American cutaneous leishmaniasis. Trans. R. Soc. Trop. Med. Hyg. 1993, 87, 444-448. 
33. Alvar, J.; Vélez, I.D.; Bern, C.; Herrero, M.; Desjeux, P.; Cano, J.; Jannin, J.; den Boer, M. WHO leishmaniasis control team, Leishmaniasis Worldwide and global estimates of its incidence. PLoS One 2012, 7, e35671.

34. Alvar, J.; Aparicio, P.; Aseffa, A.; Den Boer, M.; Cañavate, C.; Dedet, J.P.; Gradoni, L.; Ter Horst, R.; López-Vélez, R.; Moreno, J. The relationship between leishmaniasis and AIDS: the second 10 years. Clin. Microbiol. Rev. 2008, 21, 334-359.

35. Schwartz, E.; Hatz, C.; Blum, J. New world cutaneous leishmaniasis in travellers. Lancet Infect. Dis. 2006, 6, 342-349.

36. Garcia, L.S. Trypanosomiasis. In Diagnostic Medical Parasitology, 5th ed.; American Society of Microbiology: Washington, DC, USA, 2007; pp. 218-243.

37. Ives, A.; Ronet, C.; Prevel, F.; Ruzzante, G.; Fuertes-Marraco, S.; Schutz, F.; Zangger, H.; Revaz-Breton, M.; Lye, L.F.; Hickerson, S.M.; Beverley, S.M.; et al. Leishmania RNA virus controls the severity of mucocutaneous leishmaniasis. Science 2011, 331, 775-778.

38. van Griensven, J.; Diro, E. Visceral leishmaniasis. Infect. Dis. Clin. North Am. 2012, 26, 309-322.

39. Ashutosh.; Sundar, S.; Goyal, N. Molecular mechanisms of antimony resistance in Leishmania. J. Med. Microbiol. 2007, 56, 143-153.

40. Sundar, S.; Chakravarty, J. Recent advances in the diagnosis and treatment of kala-azar. Natl. Med. J. India 2012, 25, 85-89.

41. Ephros, M.; Bitnun, A.; Shaked, P.; Waldman, E.; Zilberstein, D. Stage-specific activity of pentavalent antimony against Leishmania donovani axenic amastigotes. Antimicrob. Agents Chemother. 1999, 43, 278-282.

42. Shaked-Mishan, P.; Ulrich, N.; Ephros, M.; Zilberstein, D. Novel intracellular SbV reducing activity correlates with antimony susceptibility in Leishmania donovani. J. Biol. Chem. 2001, 276, 3971-3976.

43. Singh, N.; Kumar, M.; Singh, R.K. Leishmaniasis: Current status of available drugs and new potential drug targets. Asian Pac. J. Trop. Med. 2012, 5,485-497.

44. Marquis, N.; Gourbal, B.; Rosen, B.P.; Mukhopadhyay, R.; Ouellette, M. Modulation in aquaglyceroporin AQP1 gene transcript levels in drug-resistant Leishmania. Mol. Microbiol. 2005, 57, 1690-1699.

45. Mandal, S.; Maharjan, M.; Singh, S.; Chatterjee, M.; Madhubala, R. Assessing aquaglyceroporin gene status and expression profile in antimony-susceptible and -resistant clinical isolates of Leishmania donovani from India. J. Antimicrob. Chemother. 2010, 65, 496-507.

46. Rai, S.; Bhaskar; Goel, S.K.; Nath Dwivedi, U.; Sundar, S.; Goyal, N. Role of efflux pumps and intracellular thiols in natural antimony resistant isolates of Leishmania donovani. PLoS One. 2013, 8, e74862.

47. Figarella, K.; Uzcategui, N.L.; Zhou, Y.; LeFurgey, A.; Ouellette, M.; Bhattacharjee, H.; Mukhopadhyay, R. Biochemical characterization of Leishmania major aquaglyceroporin LmAQP1: Possible role in volume regulation and osmotaxis. Mol Microbiol. 2007, 65, 1006-1017.

48. Bhattacharjee, H.; Rosen, B.P.; Mukhopadhyay, R. Aquaglyceroporins and metalloid transport: implications in human diseases. Handb. Exp. Pharmacol. 2009, 190, 309-325. 
49. Uzcategui, N.L.; Zhou, Y.; Figarella, K.; Ye, J.; Mukhopadhyay, R.; Bhattacharjee, H. Alteration in glycerol and metalloid permeability by a single mutation in the extracellular C-loop of Leishmania major aquaglyceroporin LmAQP1. Mol. Microbiol. 2008, 70, 1477-1486.

50. Mukhopadhyay, R.; Mandal, G.; Atluri, V.S.; Figarella, K.; Uzcategui, N.L.; Zhou, Y.; Beitz, E.; Ajees, A.A.; Bhattacharjee, H. The role of alanine 163 in solute permeability of Leishmania major aquaglyceroporin LmAQP1. Mol. Biochem. Parasitol. 2011, 175, 83-90.

51. Mandal, G.; Sharma, M.; Kruse, M.; Sander-Juelch, C.; Munro, L.A.; Wang, Y.; Vilg, J.V.; Tamás, M.J.; Bhattacharjee, H.; Wiese, M.; et al. Modulation of Leishmania major aquaglyceroporin activity by a mitogen-activated protein kinase. Mol. Microbiol. 2012, 85, 1204-1218.

52. Malvy, D.; Chappuis, F. Sleeping sickness. Clin. Microbiol. Infect. 2011, 17, 986-995.

53. Neglected tropical diseases. http://www.who.int/neglected_diseases/diseases/en/ (accessed on 27 November 2013).

54. Mandal, G.; Mukhopadhyay, R. The role of aquaporins in pathogenic protozoan parasites: putative target for chemotherapy. J. Med. Res. Sci. 2011, 1, 29-47.

55. Namangala, B. Contribution of innate immune responses towards resistance to African trypanosome infections. Scand J. Immunol. 2012, 75, 5-15.

56. Nok, A.J. Arsenicals (melarsoprol), pentamidine and suramin in the treatment of human African trypanosomiasis. Parasitol. Res. 2003, 90, 71-79.

57. Baker, N.; de Koning, H.P.; Mäser, P.; Horn, D. Drug resistance in African trypanosomiasis: the melarsoprol and pentamidine story. Trends Parasitol. 2013, 29,110-118.

58. Burri, C. Chemotherapy against human African trypanosomiasis: Is there a road to success? Parasitology 2010, 137, 1987-1994.

59. Mäser, P.; Lüscher, A.; Kaminsky, R. Drug transport and drug resistance in African trypanosomes. Drug Resist. Updat. 2003, 6, 281-290.

60. Bassarak, B.; Uzcátegui, N.L.; Schönfeld, C.; Duszenko, M. Functional characterization of three aquaglyceroporins from Trypanosoma brucei in osmoregulation and glycerol transport. Cell. Physiol. Biochem. 2011, 27, 411-420.

61. Baker, N.; Glover, L.; Munday, J.C.; Aguinaga Andrés, D.; Barrett, M.P.; de Koning, H.P; Horn, D. Aquaglyceroporin 2 controls susceptibility to melarsoprol and pentamidine in African trypanosomes. Proc. Natl. Acad. Sci. USA 2012, 109, 10996-11001.

62. Uzcátegui, N.L.; Figarella, K.; Bassarak, B.; Meza, N.W.; Mukhopadhyay, R.; Ramirez, J.L.; Duszenko, M. Trypanosoma brucei aquaglyceroporins facilitate the uptake of arsenite and antimonite in a pH dependent way. Cell. Physiol. Biochem. 2013, 32, 880-888.

63. Deisenhammer, F.; Bartos, A.; Egg, R.; Gilhus, N.E.; Giovannoni, G.; Rauer, S.; Sellebjerg, F. EFNS Task Force. Guidelines on routine cerebrospinal fluid analysis. Report from an EFNS task force. Eur. J. Neurol. 2006, 13, 913-922.

64. Schmidt, H.; Stuertz, K.; Chen, V.; Stringaris, A.K.; Brück, W.; Nau, R. Glycerol does not reduce neuronal damage in experimental Streptococcus pneumoniae meningitis in rabbits. Inflammopharmacology 1998, 6, 19-26.

65. Johanson, C.E.; Stopa, E.G.; McMillan, P.N. The blood-cerebrospinal fluid barrier: Structure and functional significance. Methods Mol. Biol. 2011, 686, 101-131. 
66. Edwards, A.S.; Scott, J.D. A-kinase anchoring proteins: protein kinase A and beyond. Curr. Opin. Cell Biol. 2000, 12, 217-221.

67. Luu, D.T.; Maurel, C. Aquaporin trafficking in plant cells: an emerging membrane-protein model. Traffic 2013, 14, 629-635.

68. Moeller, H.B.; Olesen, E.T.; Fenton, R.A. Regulation of the water channel aquaporin-2 by posttranslational modification. Am. J. Physiol. Renal Physiol. 2011, 300, F1062-F1073.

69. Rump, K.; Brendt, P.; Frey, U.H.; Schäfer, S.T.; Siffert, W.; Peters, J.; Adamzik, M. Aquaporin 1 and 5 expression evoked by the $\beta 2$ adrenoreceptor agonist terbutaline and lipopolysaccharide in mice and in the human monocytic cell line THP-1 is differentially regulated. Shock 2013, 40, $430-436$.

70. Morand, S.; Renggli, C.K.; Roditi, I.; Vassella, E. MAP kinase kinase 1 (MKK1) is essential for transmission of Trypanosoma brucei by Glossina morsitans. Mol. Biochem. Parasitol. 2012, 186, $73-76$.

71. Domenicali, Pfister .D.; Burkard, G.; Morand, S.; Renggli, C.K.; Roditi, I.; Vassella, E. A Mitogen-activated protein kinase controls differentiation of bloodstream forms of Trypanosoma brucei. Eukaryot. Cell 2006, 5, 1126-1135.

72. Fenn, K.; Matthews, K.R. The cell biology of Trypanosoma brucei differentiation. Curr. Opin. Microbiol. 2007, 10, 539-546.

73. Alsford, S.; Eckert, S.; Baker, N.; Glover, L.; Sanchez-Flores, A.; Leung, K.F.; Turner, D.J.; Field, M.C.; Berriman, M.; Horn, D. High-throughput decoding of antitrypanosomal drug efficacy and resistance. Nature 2012, 482, 232-236.

74. Graf, F.E.; Ludin, P.; Wenzler, T.; Kaiser, M.; Brun, R.; Pyana, P.P.; Büscher, P.; de Koning, H.P.; Horn, D.; Mäser, P. Aquaporin 2 mutations in Trypanosoma brucei gambiense field isolates correlate with decreased susceptibility to pentamidine and melarsoprol. PLoS Negl. Trop. Dis. 2013, 7, e2475.

75. Uzcátegui, N.L.; Carmona-Gutiérrez, D.; Denninger, V.; Schoenfeld, C.; Lang, F.; Figarella, K.; Duszenko, M. Antiproliferative effect of dihydroxyacetone on Trypanosoma brucei bloodstream forms: Cell cycle progression, Subcellular alterations, and cell death. Antimicrob. Agents Chemother. 2007, 51, 3960-3968.

76. Uzcátegui, N.L.; Denninger, V.; Merkel, P.; Schoenfeld, C.; Figarella, K.; Duszenko, M. Dihydroxyacetone induced autophagy in African Trypanosomes. Autophagy 2007, 3, 626-629.

77. Rassi, A., Jr.; Rassi, A.; de Rezende, J.M. American trypanosomiasis (chagas disease). Infect. Dis. Clin. North Am. 2012, 26, 275-291.

78. Kollien, A.H.; Schaub, G.A. The development of Trypanosoma cruzi in triatominae. Parasitol. Today 2000, 16, 381-387.

79. Control and prevention of Chagas disease in Europe. http://www.fac.org.ar/1/comites/chagas/ Chagas_WHO_Technical\%20Report_16_06_10.pdf(accessed on 27 November 2013).

80. Araújo, A.; Jansen, A.M.; Reinhard, K.; Ferreira, L.F. Paleoparasitology of chagas disease-a review. Mem. Inst. Oswaldo. Cruz. 2009, 104 Suppl 1, S9-S16.

81. Chagas disease (American trypanosomiasis). http://www.who.int/mediacentre/factsheets/fs340/en/ (accessed on 27 November 2013). 
82. Lescure, F.X.; Le Loup, G.; Freilij, H.; Develoux, M.; Paris, L.; Brutus, L.; Pialoux, G. Chagas disease: Changes in knowledge and management. Lancet Infect Dis. 2010, 10, 556-570.

83. Congenital Transmission of Chagas Disease - Virginia, 2010. http://www.cdc.gov/ chagas/epi.html (accessed on 27 November 2013).

84. Wilkinson, S.R.; Taylor, M.C.; Horn, D.; Kelly, J.M.; Cheeseman, I. A mechanism for cross-resistance to nifurtimox and benznidazole in trypanosomes. Proc. Natl. Acad. Sci. USA 2008, 105, 5022-5027.

85. Portal, P.; Fernández Villamil, S.; Alonso, G.D.; De Vas, M.G.; Flawiá, M.M.; Torres, H.N.; Paveto, C. Multiple NADPH-cytochrome P450 reductases from Trypanosoma cruzi suggested role on drug resistance. Mol. Biochem. Parasitol. 2008, 160, 42-51.

86. Campos, M.C.; Castro-Pinto, D.B.; Ribeiro, G.A.; Berredo-Pinho, M.M.; Gomes, L.H.; da Silva Bellieny, M.S.; Goulart, C.M.; Echevarria, A.; Leon, L.L. P-glycoprotein efflux pump plays an important role in Trypanosoma cruzi drug resistance. Parasitol. Res. 2013, 112, 2341-2351.

87. Kollien, A.H.; Grospietsch, T.; Kleffmann, T.; Zerbst-Boroffka, I.; Schaub, G.A. Ionic composition of the rectal contents and excreta of the reduviid bug Triatoma infestans. J. Insect. Physiol. 2001, 47, 739-747.

88. Docampo, R.; Moreno, S.N. Acidocalcisomes. Cell Calcium 2011, 50, 113-119.

89. Miranda, K.; de Souza, W.; Plattner, H.; Hentschel, J.; Kawazoe, U.; Fang, J.; Moreno, S.N. Acidocalcisomes in Apicomplexan parasites. Exp. Parasitol. 2008, 118, 2-9.

90. Ruiz, F.A.; Marchesini, N.; Seufferheld, M.; Govindjee; Docampo, R. The polyphosphate bodies of Chlamydomonas reinhardtii possess a proton-pumping pyrophosphatase and are similar to acidocalcisomes. J. Biol. Chem. 2001, 276, 46196-46203.

91. Marchesini, N.; Ruiz, F.A.; Vieira, M.; Docampo, R. Acidocalcisomes are functionally linked to the contractile vacuole of Dictyostelium discoideum. J. Biol. Chem. 2002, 277, 8146-8153.

92. Seufferheld, M.; Vieira, M.C.; Ruiz, F.A.; Rodrigues, C.O.; Moreno, S.N.; Docampo, R. Identification of organelles in bacteria similar to acidocalcisomes of unicellular eukaryotes. J. Biol. Chem. 2003, 278, 29971-29978.

93. Docampo, R.; Ulrich, P.; Moreno, S.N. Evolution of acidocalcisomes and their role in polyphosphate storage and osmoregulation in eukaryotic microbes. Philos. Trans. R. Soc. Lond. B Biol. Sci. 2010, 365, 775-784.

(C) 2013 by the authors; licensee MDPI, Basel, Switzerland. This article is an open access article distributed under the terms and conditions of the Creative Commons Attribution license (http://creativecommons.org/licenses/by/3.0/). 